\title{
ELEMENTARY CYCLES OF FLOWS ON MANIFOLDS
}

\author{
BY M. C. IRWIN
}

Communicated by Jurgen Moser, April 14, 1969

There are two very natural notions of equivalence of flows (see [1], [2]) on a manifold. One is the existence of a homeomorphism mapping orbits onto orbits, preserving the natural orientation of orbits but not necessarily their natural parametrisation. The second requires that the homeomorphism alter natural parametrisations by at most a positive constant multiple. We call the first relation on flows orbit-equivalence and the second flow-equivalence. There are obvious localisations of these relations. In general flow-equivalence is strictly stronger than orbit-equivalence. However, it is a consequence of the theorem of Hartman [5], [6], [7] and Grobman [3], [4] that the local notions of equivalence are the same at elementary (see [1], [2]) rest-points. The purpose of this note is to announce a similar result for elementary cycles. M. Shub has informed me that he and C. Pugh have also obtained this result.

1. Preliminaries. Let $\phi: R \times X \rightarrow X$ be a $C^{1}$ flow on a $C^{\infty}$ manifold $X$. We write $\phi_{x}(t)=\phi^{t}(x)=\phi(t, x)$, so that, for fixed $x \in X, \phi_{x}: R \rightarrow X$ is $C^{1}$ and, for fixed $t \in R, \phi^{t}: X \rightarrow X$ is a $C^{1}$ diffeomorphism. Let $U$ be open in $X$. For fixed $x \in U$ let $I_{x}$ denote the component of $\left(\phi_{x}\right)^{-1}(U)$ containing 0 , and let $D_{U}$ denote $U_{x \in U} I_{x} \times\{x\}$.

Now suppose that $\Psi$ is a $C^{1}$ flow on a $C^{\infty}$ manifold $Y$ and that $A$ and $B$ are subsets of $X$ and $Y$ respectively. We say that $A$ is flowequivalent to $B$ (with respect to the given flows) if there exist open neighbourhoods $U$ of $A$ and $V$ of $B$ and a homeomorphism $h: U \rightarrow V$ such that $h(A)=B$ and, for all $(t, x) \in D_{U}$,

$$
h \phi(t, x)=\Psi(\alpha(t), h(x)),
$$

where $\alpha: R \rightarrow R$ is a multiplication by some positive constant. In this case $h$ maps orbit components of $\phi$ in $U$ onto orbit components of $\Psi$ in $V$, preserving orientation.

Let $\nu \in G L(E)$, where $E$ is a finite dimensional real normed linear space. Let $F$ be the largest invariant subspace of $E$ on which $\nu$ has no complex eigenvalues of modulus 1 . We call $\nu \mid F$ the hyperbolic part of $\nu$.

Recall [8] that we may associate with any hyperbolic linear auto- 
morphism of a $k$-dimensional real normed linear space its suspension, which is a flow on a $(k+1)$-dimensional manifold. It has precisely one cycle, corresponding to the unique periodic point 0 of the automorphism.

2. Results. The following is an analogue, for cycles, to the theorem of Hartman and Grobman for rest-points:

Theorem 1. Let $\phi$ be a $C^{1}$ flow on $X$, and let $C$ be an elementary cycle of $\phi$. For any $x \in C$, let $D$ be the unique cycle of the suspension of the hyperbolic part of $T_{x} \phi^{\tau}$, where $\tau$ is the period of $C$. Then $C$ is flowequivalent to $D$.

As corollaries one deduces

Theorem 2. Let $C$ and $D$ be elementary cycles of $C^{1}$ flows. Then $C$ is flow-equivalent to $D$ if and only if $m(C)=m(D), n(C)=n(D)$, and $m^{-}(C)-m^{-}(D)$ and $n^{-}(C)-n^{-}(D)$ are even, where $m, n, m^{-}$and $n^{-}$ here denote the numbers of expanding, contracting, real negative expanding and real negative contracting characteristic multipliers.

and

THEOREM 3. Elementary cycles of $C^{1}$ flows are flow-equivalent if and only if they are orbit-equivalent.

The proof of Theorem 1 reduces, in effect, to a proof that there exists, at $x$, a local cross-section that is invariant under $\phi^{\tau}$. By taking a suitable chart and using a bump-function we may reduce this to the following

LEMma 4. Let $\nu: E \rightarrow E$ be a hyperbolic linear automorphism, let $f: E \rightarrow E$ be a homeomorphism and let $\zeta: E \rightarrow R$ be a linear map. Suppose that, for some $d \geqq 0, f-\nu$ and $\zeta$ vanish at 0 and on $\{x \in E ;\|x\| \geqq d\}$ and are Lipschitz, the former with constant $\kappa$. Then, if $\kappa$ is sufficiently small, there exists a continuous map $\theta: E \rightarrow R$ such that $\theta=\theta f+\zeta$.

Subject to the condition $\theta(0)=0$, the map $\theta$ is uniquely defined on the stable and unstable manifolds of the origin with respect to $f$. Elsewhere, however, it is not unique. In the proof [9] of the lemma an explicit map $\theta$ is constructed.

\section{REFERENCES}

1. R. Abraham, Foundations of mechanics, Benjamin, New York, 1967.

2. R. Abraham and J. Robbin, Transversal mappings and flows, Benjamin, New York, 1967. 
3. D. M. Grobman, Homeomorphisms of systems of differential equations, Dokl. Akad. Nauk SSSR 128 (1959), 880-881. (Russian)

4. - Topological classification of the neighborhood of a singular point in ndimensional space, Mat. Sb. 56 (98) (1962), 77-94. (Russian)

5. P. Hartman, A lemma in the theory of structural stability of differential equations, Proc. Amer. Math. Soc. 11 (1960), 610-620.

6. - On the local linearisation of differential equations, Proc. Amer. Math. Soc. 14 (1963), 568-573.

7. - Ordinary differential equations, Wiley, New York, 1964.

8. S. Smale, Differentiable dynamical systems, Bull. Amer. Soc. 73 (1967), 747-817.

9. M. C. Irwin, A classification of elementary cycles, Topology (to appear).

University of Liverpool, Liverpool, ENGLAND 